\title{
Subspace Lattices, Reflexivity and Tensor Products
}

\author{
IVAN G. TODOROV
}

\begin{abstract}
This article surveys results on reflexivity of subspace lattices and their tensor products. The lattice tensor product formula is addressed and one new result in this direction is included.
\end{abstract}

\section{INTRODUCTION}

The Invariant Subspace Problem is nowadays recognised as one of the most important open problems in Operator Theory. It asks whether every bounded linear operator acting on a Hilbert space possesses an invariant subspace different from $\{0\}$ and the whole space. For a Hilbert space $\mathcal{H}$, let $\mathcal{B}(\mathcal{H})$ be the collection of all bounded linear operators on $\mathcal{H}, \mathcal{S}(\mathcal{H})$ be the collection of all closed subspaces of $\mathcal{H}$ and $\mathcal{P}(\mathcal{H})$ be the collection of all orthogonal projections on $\mathcal{H}$. The set $\mathcal{S}(\mathcal{H})$ is a complete lattice under the operations of intersection and closed linear span. The one-to-one correspondence between closed subspaces and orthogonal projections allows us to transfer the lattice structure of $\mathcal{S}(\mathcal{H})$ to $\mathcal{P}(\mathcal{H})$, thus turning $\mathcal{P}(\mathcal{H})$ into a complete lattice. If $\mathcal{A} \subseteq \mathcal{B}(\mathcal{H})$ is any family then the set

$$
\text { lat } \mathcal{A}=\{P \in \mathcal{P}(\mathcal{H}): A P=P A P \text {, for each } A \in \mathcal{A}\}
$$

is easily seen to be a complete sublattice of $\mathcal{P}(\mathcal{H})$; it is called the invariant subspace lattice of $\mathcal{A}$ since the ranges of the projections in lat $\mathcal{A}$ are precisely the closed $\mathcal{A}$-invariant subspaces. The Invariant Subspace Problem can now be restated as the question of whether there exists an operator $A \in \mathcal{B}(\mathcal{H})$ such that lat $\{A\}=\{0, I\}$.

Clearly, the last formulation is a special case of two more general problems.

Problem 1 Given a set $\mathcal{A} \subseteq \mathcal{B}(\mathcal{H})$, determine the lattice lat $\mathcal{A}$.

Problem 2 Given a lattice $\mathcal{L} \subseteq \mathcal{P}(\mathcal{H})$, determine the subsets $\mathcal{A} \subseteq$ $\mathcal{B}(\mathcal{H})$ for which lat $\mathcal{A}=\mathcal{L}$. 
These problems are inverse to one another. Problem 1 can be thought of as a problem of analysis, while Problem 2 - as a problem of synthesis. We will give later a more rigorous meaning to this interpretation. For the time being, note that if $\tilde{\mathcal{A}}$ is the weakly closed subalgebra of $\mathcal{B}(\mathcal{H})$ generated by a set $\mathcal{A} \subseteq \mathcal{B}(\mathcal{H})$ then lat $\mathcal{A}=$ lat $\tilde{\mathcal{A}}$. We may thus assume that our families $\mathcal{A}$ are subalgebras of $\mathcal{B}(\mathcal{H})$ closed in the weak operator topology. ${ }^{1}$ It is trivial to check that an invariant subspace lattice is automatically strongly closed. We may thus assume that our sublattices $\mathcal{L} \subseteq \mathcal{P}(\mathcal{H})$ are closed in the strong operator topology. Such lattices are traditionally called subspace lattices. The subspace lattices $\mathcal{L}$ for which there exists $\mathcal{A}$ with lat $\mathcal{A}=\mathcal{L}$ are called reflexive.

Problem 2 includes the question of deciding which subspace lattices are reflexive. This question has attracted much attention since Halmos' foundational paper [4]; many of its aspects are at present widely open. In this, mainly survey, article, the milestones of this investigation will be described. One of the most important contributions in this direction is Arveson's theorem for the reflexivity of commutative subspace lattices [1]. This result will be discussed and other classes of reflexive lattices will be exhibited. The question of how to build new reflexive lattices from already existing ones will be addressed. This will lead naturally to the introduction of tensor products and the discussion of the problem known as the "lattice tensor product formula" [6]. The known cases for which this formula holds will be described and a new one will be added to that list. Open problems will be discussed throughout the exposition. Since we will be often dealing with measure theoretic constructions, we will assume throughout that our Hilbert spaces are separable.

\section{Classes of Subspace Lattices}

Subspace lattices first appeared in the 1930's in von Neumann's work on the mathematical foundations of quantum mechanics. A von Neumann algebra is a weakly closed selfadjoint subalgebra of $\mathcal{B}(\mathcal{H})$. If $\mathcal{A}$ is a von Neumann algebra then the collection $\mathcal{L}$ of the non-trivial projections belonging to $\mathcal{A}$ is non-empty: the spectral measure of every selfadjoint operator $A \in \mathcal{A}$ is entirely contained

\footnotetext{
${ }^{1} \mathrm{~A}$ net $\left\{A_{i}\right\}$ converges to $A$ in the weak operator topology if $\left(A_{i} x, y\right) \rightarrow$ $(A x, y)$ for all $x, y \in \mathcal{H}$, and it converges to $A$ in the strong operator topology if $A_{i} x \rightarrow A x$ for all $x \in \mathcal{H}$.
} 
in $\mathcal{A}$. As a matter of fact, $\mathcal{L}$ is reflexive: it coincides with the invariant subspace lattice of the commutant $\mathcal{A}^{\prime}$ of $\mathcal{A}$. Indeed, if $L \in \mathcal{L}$ and $A \in \mathcal{A}^{\prime}$ then $L^{\perp} A L=L^{\perp} L A=0$, so $\mathcal{L} \subseteq$ lat $\mathcal{A}^{\prime}$. Conversely, suppose that $M \in$ lat $\mathcal{A}^{\prime}$ and $A \in \mathcal{A}^{\prime}$. Then $A M=M A M$. Since $\mathcal{A}^{\prime}$ is selfadjoint, we also have $A^{*} M=M A^{*} M$. These two identities give $A M=M A$, therefore $M \in \mathcal{A}^{\prime \prime}$. By von Neumann's Bicommutant Theorem, $\mathcal{A}^{\prime \prime}=\mathcal{A}$ and so $M \in \mathcal{L}$. Call the projection lattice of a von Neumann algebra a von Neumann lattice. Von Neumann lattices are orthocomplemented; using the Bicommutant Theorem once again we can see that an orthocomplemented lattice is reflexive precisely when it is a von Neumann lattice.

The subspace lattices, structurally opposite to von Neumann lattices, are the totally ordered ones, also called nests. They were introduced by Ringrose in [11], where he showed that every nest is reflexive. The idea behind the proof is the following: if $\mathcal{N}$ is a nest, let $\mathcal{A}_{0}$ be the weak closure of the subspace generated by the "full corners" $\mathcal{B}\left(M^{\perp} \mathcal{H}, N \mathcal{H}\right)$, where $N, M \in \mathcal{N}, N<M$. Then one can show that lat $\mathcal{A}_{0}=\mathcal{N}$.

Note that every nest $\mathcal{L}$ is a commutative subspace lattice (CSL), in other words, any two projections $P, Q \in \mathcal{L}$ satisfy the relation $P Q=Q P$. This property was taken as a starting point by Arveson in [1], where he established the following fundamental result.

Theorem 2.1. Every commutative subspace lattice is reflexive.

Before describing Arveson's approach to the problem, we consider a special case. Let $\mathcal{L}$ be a CSL. An atom of $\mathcal{L}$ is a projection $E$ (not necessarily belonging to $\mathcal{L}$ ) such that, for any $L \in \mathcal{L}$, either $E \leq L$ or $E L=0$. If the identity projection $I$ is the span of all atoms of $\mathcal{L}$ then $\mathcal{L}$ is called totally atomic. Fix a totally atomic CSL $\mathcal{L}$, acting on a Hilbert space $\mathcal{H}$, and let at $(\mathcal{L})$ be the set of all its atoms. It is easily seen that if $L \in \mathcal{L}$ then $L=\vee\{E \in$ at $(\mathcal{L}): E \leq L\}$. For each $E \in \operatorname{at}(\mathcal{L})$ let

$$
\gamma(E)=\wedge\{L \in \mathcal{L}: E \leq L\} .
$$

Introduce a binary relation $\prec$ on at $(\mathcal{L})$ by letting $E \prec F$ if $\gamma(F) \leq$ $\gamma(E)$. One readily verifies that $\prec$ is a partial order. Call a subset $\alpha \subseteq$ at $(\mathcal{L})$ increasing if $E \prec F$ and $E \in \alpha$ imply $F \in \alpha$. Let $\mathcal{L}_{0}$ be the collection of all projections of the form $\vee \alpha$, where $\alpha \subseteq$ at $(\mathcal{L})$ is increasing, and $\mathcal{A}_{0}$ be the algebra generated by the subspaces $F \mathcal{B}(\mathcal{H}) E$, with $E \prec F$. We first observe that lat $\mathcal{A}_{0}=\mathcal{L}_{0}$. Indeed, 
let $\alpha \subseteq$ at $(\mathcal{L})$ be increasing and $L=\vee \alpha$. Fix $E, F \in$ at $(\mathcal{L})$ with $E \prec F$. If $E \in \alpha$ then $F \in \alpha$, so $F \leq L$ and $L^{\perp} F \mathcal{B}(\mathcal{H}) E L=\{0\}$. If $E \notin \alpha$ then $E L=0$ and again $L^{\perp} F \mathcal{B}(\mathcal{H}) E L=\{0\}$. Thus, $\mathcal{L}_{0} \subseteq$ lat $\mathcal{A}_{0}$.

Given a projection $L$ on $\mathcal{H}$ define $\alpha(L)=\{E \in$ at $(\mathcal{L}): E \leq L\}$. Assume that $L \in$ lat $\mathcal{A}_{0}$ and let $\alpha=\alpha(L)$. For each $E \in$ at $(\mathcal{L})$, the space $E \mathcal{B}(\mathcal{H}) E$ is contained in $\mathcal{A}_{0}$. A few moments' thought now shows that $L=\vee \alpha$. Suppose that $E \in \alpha$ and $E \prec F$. Then

$$
\overline{[(F \mathcal{B}(\mathcal{H}) E) L \mathcal{H}]}=\overline{[F \mathcal{B}(\mathcal{H}) E \mathcal{H}]}=F \mathcal{H}
$$

and since $L$ is invariant for $\mathcal{A}_{0}$, we conclude that $F \in \alpha$. It follows that $\alpha$ is increasing.

We thus have lat $\mathcal{A}_{0}=\mathcal{L}_{0}$ and therefore to show that $\mathcal{L}$ is reflexive it suffices to establish the following

Claim $\mathcal{L}=\mathcal{L}_{0}$.

Proof. First note that if $L \in \mathcal{L}$ then $\alpha(L)$ is increasing. Indeed, suppose $E \in \alpha(L)$; this yields $\gamma(E) \leq L$. If $E \prec F$ then $\gamma(F) \leq \gamma(E)$ and so $\gamma(F) \leq L$ which means that $F \leq L$ and so $F \in \alpha(L)$. Since $L=\vee \alpha(L)$, we conclude that $\mathcal{L} \subseteq \mathcal{L}_{0}$.

Conversely, let $\alpha \subseteq$ at $(\mathcal{L})$ be increasing. For each $E \in \alpha$ we have

$$
\begin{aligned}
\gamma(E) & =\bigvee\{G \in \operatorname{at}(\mathcal{L}): G \leq \gamma(E)\} \\
& =\bigvee\{G \in \operatorname{at}(\mathcal{L}): \gamma(G) \leq \gamma(E)\}=\bigvee\{G \in \operatorname{at}(\mathcal{L}): E \prec G\} .
\end{aligned}
$$

It follows that $\vee \alpha=\bigvee\{\gamma(E): E \in \alpha\} \in \mathcal{L}$ and so $\mathcal{L}_{0} \subseteq \mathcal{L}$.

Thus, every totally atomic CSL is reflexive. The above proof depends heavily on the atomicity of the lattice. If one wishes to generalise the argument to arbitrary CSL's, she or he should first find an appropriate substitute for at $(\mathcal{L})$. It was Arveson's idea that this substitute could be a measure space, whose points play the role of the atoms of $\mathcal{L}$, in case $\mathcal{L}$ has no atoms. To be more concrete, fix a standard (finite) measure space $(X, \mu)$. Let $\prec$ be a standard quasiorder on $X$, that is, a reflexive and transitive binary relation, for which there exist countably many measurable functions $f: X \rightarrow \mathbb{R}$ such that $x \prec y$ if and only if $f_{n}(x) \leq f_{n}(y)$, for each $n \in \mathbb{N}$. Call a measurable set $\alpha \subseteq X$ increasing if there exists a null set $M \subset X$ such that $x, y \notin M, x \in \alpha$ and $x \prec y$ imply $y \in \alpha$. On the Hilbert space $\mathcal{H}=L^{2}(X, \mu)$ consider the collection of projections

$$
\mathcal{L}(X, \prec)=\{P(\alpha): \alpha \text { increasing }\},
$$


where $P(\alpha)$ is the multiplication by the characteristic function of $\alpha$. Since the family of increasing subsets of $X$ is closed under finite unions and intersections, $\mathcal{L}(X, \prec)$ is a lattice. A bit more work is required to show that $\mathcal{L}(X, \prec)$ is strongly closed, but it is true. Since $P(\alpha) P(\beta)=P(\alpha \cap \beta)$, the elements of $\mathcal{L}(X, \prec)$ commute with each other. We conclude that $\mathcal{L}(X, \prec)$ is a CSL. Arveson showed in [1] that every CSL is unitarily equivalent to $\mathcal{L}(X, \prec)$, for an appropriate choice of a measure space $X$ and a standard quasi-order $\prec$. This "Spectral Theorem for CSL's" is the statement corresponding to the Claim in the case of a general lattice. Arveson also exhibited an appropriate substitute $\mathcal{A}_{0}(X, \prec)$ for the algebra $\mathcal{A}_{0}$ in the argument above, defined as the closure of a family of "pseudointegral" operators which live in the graph of $\prec$, in a similar way as the operators $T \in \mathcal{A}_{0}$ live in the spaces $\mathcal{B}(F \mathcal{H}, E \mathcal{H})$ with $F \prec E$. He established the reflexivity of $\mathcal{L}(X, \prec)$ by showing that $\mathcal{L}(X, \prec)=$ lat $\mathcal{A}_{0}(X, \prec)$.

\section{Generating New Reflexive Lattices}

It is not only a question of philosophical nature in mathematics to study whether a property of interest is preserved under certain operations. Starting with an object or objects possessing a given property, this often allows to construct new objects with the same property. For subspace lattices, a natural operation is forming the join of two (or finitely many) lattices. Given subspace lattices $\mathcal{L}_{1}$ and $\mathcal{L}_{2}$, by $\mathcal{L}_{1} \vee \mathcal{L}_{2}$ we denote the subspace lattice generated by $\mathcal{L}_{1}$ and $\mathcal{L}_{2}$. The reflexivity of $\mathcal{L}_{1}$ and $\mathcal{L}_{2}$ does not imply that of $\mathcal{L}_{1} \vee \mathcal{L}_{2}$. Indeed, in [10] it is shown that there exist non-reflexive lattices of the form $\mathcal{L}=\{0, L, M, N, I\}$, where $M \leq N, L \wedge N=0$ and $L \vee M=I$. Clearly, $\mathcal{L}$ is the join of the nests $\mathcal{L}_{1}=\{0, M, N, I\}$ and $\mathcal{L}_{2}=\{0, L, I\}$.

Question 3.1. Suppose $\mathcal{L}_{1}$ and $\mathcal{L}_{2}$ are reflexive lattices acting on the same Hilbert space. When is $\mathcal{L}_{1} \vee \mathcal{L}_{2}$ reflexive?

In concrete situations, some restrictions on the mutual position of $\mathcal{L}_{1}$ and $\mathcal{L}_{2}$ are imposed e.g. that $\mathcal{L}_{1}$ and $\mathcal{L}_{2}$ commute with each other. The reason is that, without such an assumption on the generating lattices, very little can be said about the generated lattice. We gather some important contributions to the study of Question 3.1 in the following theorem. 
Theorem 3.2. Let $\mathcal{L}_{1}$ and $\mathcal{L}_{2}$ be subspace lattices acting on the same Hilbert space and commuting with each other. The lattice $\mathcal{L}_{1} \vee \mathcal{L}_{2}$ is reflexive whenever one of the following properties holds:

(i) $\mathcal{L}_{1}$ is a nest and $\mathcal{L}_{2}$ is a von Neumann lattice;

(ii) $\mathcal{L}_{1}$ is a totally atomic $C S L$ and $\mathcal{L}_{2}$ is a reflexive lattice;

(iii) $\mathcal{L}_{1}$ is a CSL and $\mathcal{L}_{2}$ is a reflexive lattice containing the projection lattice of a properly infinite von Neumann algebra.

Part (i) in the above theorem is due to Gilfeather and Larson [3]. Part (ii) and (iii) are due to Katsoulis [8] in the case $\mathcal{L}_{2}$ is a von Neumann lattice and to Symes [13] in the general case.

To be able to say more about the structure of $\mathcal{L}_{1} \vee \mathcal{L}_{2}$, one should assume that $\mathcal{L}_{1}$ and $\mathcal{L}_{2}$ are in even nicer position than that of each one being contained in the commutant of the other. This is where tensor products should be introduced. Let $\mathcal{H}_{1}$ and $\mathcal{H}_{2}$ be Hilbert spaces and $\mathcal{H}=\mathcal{H}_{1} \otimes \mathcal{H}_{2}$ be the Hilbert space tensor product of $\mathcal{H}_{1}$ and $\mathcal{H}_{2}$. If $A \in \mathcal{B}\left(\mathcal{H}_{1}\right)$ and $B \in \mathcal{B}\left(\mathcal{H}_{2}\right)$ then the equality

$$
(A \otimes B)(\xi \otimes \eta)=A \xi \otimes B \eta \quad\left(\xi \in \mathcal{H}_{1}, \eta \in \mathcal{H}_{2}\right)
$$

defines a bounded linear operator on $\mathcal{H}$. If $P \in \mathcal{P}\left(\mathcal{H}_{1}\right)$ and $Q \in$ $\mathcal{P}\left(\mathcal{H}_{2}\right)$ then $P \otimes Q \in \mathcal{P}(\mathcal{H})$. Thus, given subspace lattices $\mathcal{L}_{1}$ and $\mathcal{L}_{2}$ on $\mathcal{H}_{1}$ and $\mathcal{H}_{2}$, respectively, we can form the subspace lattice $\mathcal{L}=\mathcal{L}_{1} \otimes \mathcal{L}_{2}$ generated by the elementary tensors $P \otimes Q$, where $P \in \mathcal{L}_{1}$ and $Q \in \mathcal{L}_{2}$. Note that $\mathcal{L}$ is the subspace lattice generated by the amplifications $\mathcal{L}_{1} \otimes \mathbf{1}$ and $\mathbf{1} \otimes \mathcal{L}_{2}$ of $\mathcal{L}_{1}$ and $\mathcal{L}_{2}$. (For a subspace lattice $\mathcal{M}$ we write $\mathcal{M} \otimes \mathbf{1}=\{M \otimes I: M \in \mathcal{M}\}$.) Of course, $\mathcal{L}_{1} \otimes \mathbf{1}$ and $\mathbf{1} \otimes \mathcal{L}_{2}$ commute with each other. Our initial question can now be specialised:

Question 3.3. Suppose $\mathcal{L}_{1}$ and $\mathcal{L}_{2}$ are reflexive lattices. When is $\mathcal{L}_{1} \otimes \mathcal{L}_{2}$ reflexive?

The assumption in Question 3.3 is that $\mathcal{L}_{i}=$ lat $\mathcal{A}_{i}, i=1,2$, for some operator algebras $\mathcal{A}_{1}$ and $\mathcal{A}_{2}$, and one wants to determine whether there exists an operator algebra $\mathcal{A}$ such that $\mathcal{L}_{1} \otimes \mathcal{L}_{2}=$ lat $\mathcal{A}$. There is a natural candidate for $\mathcal{A}$, namely the (weakly closed) algebra $\mathcal{A}_{1} \otimes \mathcal{A}_{2}$ generated by the elementary tensors $A \otimes B$, where $A \in \mathcal{A}_{1}$ and $B \in \mathcal{A}_{2}$. We thus arrive at a more special question:

Question 3.4. Suppose $\mathcal{A}_{1} \subseteq \mathcal{B}\left(\mathcal{H}_{1}\right)$ and $\mathcal{A}_{2} \subseteq \mathcal{B}\left(\mathcal{H}_{2}\right)$ are operator algebras. When does the formula below hold

$$
\operatorname{lat}\left(\mathcal{A}_{1} \otimes \mathcal{A}_{2}\right)=\operatorname{lat} \mathcal{A}_{1} \otimes \operatorname{lat} \mathcal{A}_{2} ?
$$


Equation (1) is called the lattice tensor product formula (LTPF). Its investigation was initiated by Hopenwasser in [6], as a "dual" problem to an older question, the algebra tensor product formula (ATPF). The cautious reader will have noticed that there is a natural "inverse" construction to that of passing from an algebra to its invariant subspace lattice. Namely, if $\mathcal{L}$ is a subspace lattice, one can define

$$
\operatorname{alg} \mathcal{L}=\{A \in \mathcal{B}(\mathcal{H}): A L=L A L, \text { for each } L \in \mathcal{L}\},
$$

the algebra of all operators leaving invariant the projections of $\mathcal{L}$. ATPF is the identity

$$
\operatorname{alg}\left(\mathcal{L}_{1} \otimes \mathcal{L}_{2}\right)=\operatorname{alg} \mathcal{L}_{1} \otimes \operatorname{alg} \mathcal{L}_{2} .
$$

ATPF was first studied in [2]; although not universally true [9], it has been established in a number of cases. However, it is still open for CSL's. If $\mathcal{L}_{1}$ and $\mathcal{L}_{2}$ are von Neumann lattices and $\mathcal{R}_{i}=\mathcal{L}_{i}^{\prime \prime}$, $i=1,2$, the ATPF is equivalent to the identity

$$
\left(\mathcal{R}_{1} \otimes \mathcal{R}_{2}\right)^{\prime}=\mathcal{R}_{1}^{\prime} \otimes \mathcal{R}_{2}^{\prime}
$$

This is the well known Tomita Commutation Theorem, a fundamental result in von Neumann algebra theory. Its lattice version, the LTPF for von Neumann algebras, is open. It amounts to verifying that, if $\mathcal{L}_{1}$ and $\mathcal{L}_{2}$ are von Neumann lattices then $\mathcal{L}_{1} \otimes \mathcal{L}_{2}$ is also a von Neumann lattice. In [6] this was shown to hold if $\mathcal{L}_{1}$ and $\mathcal{L}_{2}$ are injective von Neumann lattices (that is, projection lattices of injective von Neumann algebras), while in [12] it was established in the case only one of the von Neumann lattices is injective.

A special case of LTPF is obtained when $\mathcal{A}_{2}$ is the scalar multiples of the identity on $\mathcal{H}_{2}$ and $\operatorname{dim} \mathcal{H}_{2}=\infty$. Let $\mathcal{P}=\mathcal{P}\left(\mathcal{H}_{2}\right)$. Validity of LTPF is in this case equivalent to the reflexivity of lat $\mathcal{A}_{1} \otimes \mathcal{P}$. Say that a (not necessarily reflexive) subspace lattice $\mathcal{L}$ possesses property (p) if $\mathcal{L} \otimes \mathcal{P}$ is reflexive.

Question 3.5. Does there exist a subspace lattice without property $(p)$ ?

Lattices with property $(\mathrm{p})$ were exhibited in [12]: such are the lattices of the form $(\mathcal{L} \vee \mathcal{N}) \otimes \mathcal{N}_{0}$, where $\mathcal{L}$ is a CSL, $\mathcal{N}$ and $\mathcal{N}_{0}$ are von Neumann lattices, at least one of which is injective, and $\mathcal{N}$ commutes with $\mathcal{L}$. In particular, every CSL possesses property (p). If $\mathcal{P}$ is replaced by the lattice $\mathcal{P}_{n}$ of all projections on an $n$-dimensional 
Hilbert space, with $n<\infty$, the corresponding question, i.e., the question of whether $\mathcal{L} \otimes \mathcal{P}_{n}$ is reflexive, is open.

\section{Tensoring With A CSL}

Finding a convenient description of a tensor product lattice is crucial for deciding whether it is reflexive. If one of the lattices is a CSL, Arveson's coordinates discussed in Section 2 are very useful. Suppose that $\mathcal{L}=\mathcal{L}(X, \prec)$ is the CSL corresponding to a standard quasi-ordered measure space $(X, \prec, \mu)$ acting on $\mathcal{H}_{0}=L^{2}(X, \mu)$. Let $\mathcal{N}$ be a subspace lattice acting on a Hilbert space $\mathcal{K}$. The Hilbert space $\mathcal{H}=\mathcal{H}_{0} \otimes \mathcal{K}$ can be identified with the collection $L^{2}(X, \mathcal{K})$ of all square integrable $\mathcal{K}$-valued functions on $X$. Each element $\varphi \in L^{\infty}(X, \mu)$ gives rise to a multiplication operator on $\mathcal{H}_{0}: M_{\varphi} f=\varphi f$. The collection $\mathcal{D}$ of all such operators is a maximal abelian selfadjoint algebra (masa) on $\mathcal{H}_{0}$ and $\mathcal{D} \otimes \mathcal{B}(\mathcal{K})$ can be identified with the family $L^{\infty}(X, \mathcal{B}(\mathcal{K}), \mu)$ of all (weakly) measurable essentially bounded functions $A: X \rightarrow \mathcal{B}(\mathcal{K})$ acting on $\mathcal{H}$ pointwise. If $\varphi \in L^{\infty}(X, \mu)$ and $B \in \mathcal{B}(\mathcal{K})$ then $M_{\varphi} \otimes B$ corresponds to the function $\varphi B$. Under this identification, $\mathcal{P}(\mathcal{D} \otimes \mathcal{B}(\mathcal{K}))$ consists of all projection valued (weakly measurable) functions on $X$. If $P(\alpha) \in \mathcal{L}$ and $Q \in \mathcal{N}$ then the function $P$ corresponding to $P(\alpha) \otimes Q$ is given by $P(x)=Q$ if $x \in \alpha$ and $P(x)=0$ otherwise. The function $P$ is moreover increasing: on a set of full measure, $x \prec y$ implies $P(x) \leq P(y)$. On the other hand, the set $\mathcal{L}(X, \prec, \mathcal{N})$ of all increasing $\mathcal{N}$-valued functions on $X$ is easily seen to be a subspace lattice. It follows that

$$
\mathcal{L} \otimes \mathcal{N} \subseteq \mathcal{L}(X, \prec, \mathcal{N}) .
$$

The lattice $\mathcal{L}(X, \prec, \mathcal{N})$ is reflexive whenever $\mathcal{N}$ is reflexive: if $\mathcal{N}=$ lat $\mathcal{B}$ then $\left.\mathcal{L}(X, \prec, \mathcal{N})=\operatorname{lat}\left(\mathcal{A}_{0}(X, \prec)\right) \otimes \mathcal{B}\right)$. Thus, to show that $\mathcal{L} \otimes \mathcal{N}$ is reflexive, it suffices to prove that equality holds in (3). This observation was the basis for establishing LTPF if lat $\mathcal{A}_{1}$ is a totally atomic CSL or a nest and $\mathcal{A}_{2}$ is the scalar multiples of the identity in [6], and if lat $\mathcal{A}_{1}$ is a completely distributive $\mathrm{CSL}^{2}$ or a Boolean lattice and $\mathcal{A}_{2}$ is arbitrary in [5].

If $\mathcal{L}$ is a CSL and $\mathcal{A}=\operatorname{alg} \mathcal{L}$ then any masa containing $\mathcal{L}$ is easily seen to be contained in $\mathcal{A}$. Much as in Problem 2, we may look at

\footnotetext{
${ }^{2}$ Complete distributivity is the validity of the distributivity laws for arbitrary subsets of the lattice.
} 
the family

$$
\begin{aligned}
\mathbb{A}(\mathcal{L})=\{\mathcal{B} \subseteq \mathcal{B}(\mathcal{H}) & \text { : } \mathrm{w}^{*} \text {-closed algebra } \\
& \text { containing masa with lat } \mathcal{B}=\mathcal{L}\} .
\end{aligned}
$$

Theorem 2.1 implies that $\operatorname{alg} \mathcal{L}$ is the maximal element of $\mathbb{A}(\mathcal{L})$. Arveson showed [1] that the algebra $\mathcal{A}_{0}$ associated to $\mathcal{L}$ as discussed in Section 2 is the minimal element of $\mathbb{A}(\mathcal{L})$. The lattice $\mathcal{L}$ is called synthetic if $\mathcal{A}_{0}=\operatorname{alg} \mathcal{L}$ [1]. We formulate a question for which the above description of the tensor product is relevant; it is a special case of Question 3.3.

Question 4.1. Let $\mathcal{L}$ be a CSL and $\mathcal{N}$ be a von Neumann lattice. Is $\mathcal{L} \otimes \mathcal{N}$ reflexive? What if $\mathcal{L}$ is additionally assumed to be synthetic?

We finally establish LTPF in one more case. The algebras belonging to some of the classes $\mathbb{A}(\mathcal{L})$ will be called Arveson algebras. For an operator algebra $\mathcal{A}$ we denote by $\mathcal{P}(\mathcal{A})$ the collection of all projections in $\mathcal{A}$.

Theorem 4.2. Let $\mathcal{A}_{1}$ and $\mathcal{A}_{2}$ be Arveson algebras. Then

$$
\operatorname{lat}\left(\mathcal{A}_{1} \otimes \mathcal{A}_{2}\right)=\text { lat } \mathcal{A}_{1} \otimes \text { lat } \mathcal{A}_{2} \text {. }
$$

Proof. According to our previous discussion, we may assume that $\mathcal{A}_{i}$ acts on $\mathcal{H}_{i}=L^{2}\left(X_{i}, \mu_{i}\right)$, contains the masa $\mathcal{D}_{i} \equiv L^{\infty}\left(X_{i}, \mu_{i}\right)$ and $\mathcal{L}_{i}=$ lat $\mathcal{A}_{i}=\mathcal{L}\left(X_{i}, \prec_{i}\right)$, for some standard quasi-order $\prec_{i}, i=1,2$. We have $\mathcal{H}_{1} \otimes \mathcal{H}_{2}=L^{2}\left(X_{1} \times X_{2}, \mu_{1} \times \mu_{2}\right)$. It is shown in [2] that

$$
\mathcal{L} \stackrel{\text { def }}{=} \mathcal{L}_{1} \otimes \mathcal{L}_{2}=\mathcal{L}\left(X_{1} \times X_{2}, \prec\right),
$$

where $\prec$ is the product order on $X_{1} \times X_{2}{ }^{3}$ Let $\mathcal{S}_{i}=$ lat $\mathcal{D}_{i}, i=1,2$. Then $\mathcal{S}_{i}$ is a Boolean lattice coinciding with $\mathcal{P}\left(\mathcal{D}_{i}\right)$. We first note that

$$
\operatorname{lat}\left(\mathcal{A}_{1} \otimes \mathcal{A}_{2}\right)=\left(\mathcal{L}_{1} \otimes \mathcal{S}_{2}\right) \cap\left(\mathcal{S}_{1} \otimes \mathcal{L}_{2}\right) .
$$

Indeed, $\mathcal{A}_{1} \otimes \mathcal{D}_{2} \subseteq \mathcal{A}_{1} \otimes \mathcal{A}_{2}$ and, since LTPF holds if one of the lattices is Boolean,

$$
\operatorname{lat}\left(\mathcal{A}_{1} \otimes \mathcal{A}_{2}\right) \subseteq \operatorname{lat}\left(\mathcal{A}_{1} \otimes \mathcal{D}_{2}\right)=\mathcal{L}_{1} \otimes \mathcal{S}_{2} .
$$

Similarly, lat $\left(\mathcal{A}_{1} \otimes \mathcal{A}_{2}\right) \subseteq \mathcal{S}_{1} \otimes \mathcal{L}_{2}$. Thus

$$
\operatorname{lat}(\mathcal{A} \otimes \mathcal{B}) \subseteq\left(\mathcal{L}_{1} \otimes \mathcal{S}_{2}\right) \cap\left(\mathcal{S}_{1} \otimes \mathcal{L}_{2}\right) .
$$

\footnotetext{
${ }^{3}$ By definition, $\left(x_{1}, x_{2}\right) \prec\left(y_{1}, y_{2}\right)$ if $x_{1} \prec 1 y_{1}$ and $x_{2} \prec y_{2}$.
} 
Conversely, let $P \in\left(\mathcal{L}_{1} \otimes \mathcal{S}_{2}\right) \cap\left(\mathcal{S}_{1} \otimes \mathcal{L}_{2}\right)$. Then the projection $P$ is invariant for $\mathcal{A}_{1} \otimes \mathcal{D}_{2}$ and for $\mathcal{D}_{1} \otimes \mathcal{A}_{2}$. If $A \in \mathcal{A}_{1}$ and $B \in \mathcal{A}_{2}$ we have

$(A \otimes B) P=(A \otimes I)(I \otimes B) P=(A \otimes I) P(I \otimes B) P=P(A \otimes I) P(I \otimes B) P$ and thus $P$ is invariant for $A \otimes B$. Since $\mathcal{A}_{1} \otimes \mathcal{A}_{2}$ is generated by the elementary tensors, we conclude that $P$ is invariant for $\mathcal{A}_{1} \otimes \mathcal{A}_{2}$.

Now let $P \in \operatorname{lat}\left(\mathcal{A}_{1} \otimes \mathcal{A}_{2}\right)$. Then $P \in \operatorname{lat}\left(\mathcal{D}_{1} \otimes \mathcal{D}_{2}\right)=\mathcal{P}\left(L^{\infty}\left(X_{1} \times\right.\right.$ $\left.X_{2}, \mu_{1} \times \mu_{2}\right)$ ) and hence $P=P(E)$ is the multiplication by the characteristic function of some measurable set $E \subseteq X_{1} \times X_{2}$. By (5), $P \in \mathcal{L}_{1} \otimes \mathcal{S}_{2}$ and since $\mathcal{S}_{2}$ corresponds to the trivial order on $X_{2}$ ( $x$ and $y$ are comparable only when $x=y$ ), it follows from (4) that $E$ is increasing with respect to the first variable: there exists a null set $M \subseteq X_{1} \times X_{2}$ such that

$$
\left(x_{1}, z\right),\left(y_{1}, z\right) \notin M,\left(x_{1}, z\right) \in E \text { and } x_{1} \prec_{1} y_{1} \text { imply }\left(y_{1}, z\right) \in E \text {. }
$$

Similarly, there exists a null set $N \subseteq X_{1} \times X_{2}$ such that

$$
\left(z, x_{2}\right),\left(z, y_{2}\right) \notin N,\left(z, x_{2}\right) \in E \text { and } x_{2} \prec_{2} y_{2} \text { imply }\left(z, y_{2}\right) \in E \text {. }
$$

Now suppose that $\left(x_{1}, x_{2}\right),\left(y_{1}, y_{2}\right) \notin M \cup N,\left(x_{1}, x_{2}\right) \in E$ and $\left(x_{1}, x_{2}\right) \prec\left(y_{1}, y_{2}\right)$. Then $x_{1} \prec_{1} y_{1}$ and $x_{2} \prec_{2} y_{2}$. By $(6),\left(y_{1}, x_{2}\right) \in E$ and now by $(7),\left(y_{1}, y_{2}\right) \in E$. Thus, $E$ is $\prec$-increasing and so $P \in \mathcal{L}$.

As in the proof of Theorem 4.2, it can be seen that if $\mathcal{A}_{1}$ and $\mathcal{A}_{2}$ are algebras for which $\mathcal{L}_{1}=$ lat $\mathcal{A}_{1}$ and $\mathcal{L}_{2}=$ lat $\mathcal{A}_{2}$ possess property (p) then

$$
\operatorname{lat}\left(\mathcal{A}_{1} \otimes \mathcal{A}_{2}\right)=\left(\mathcal{L}_{1} \otimes \mathcal{P}\right) \cap\left(\mathcal{P} \otimes \mathcal{L}_{2}\right) .
$$

Thus, in this case, LTPF is equivalent to the identity

$$
\mathcal{L}_{1} \otimes \mathcal{L}_{2}=\left(\mathcal{L}_{1} \otimes \mathcal{P}\right) \cap\left(\mathcal{P} \otimes \mathcal{L}_{2}\right) .
$$

Question 4.3. When does identity (8) hold?

We finish with a corollary of Theorem 4.2.

Corollary 4.4. Let $\mathcal{L}_{1}$ and $\mathcal{L}_{2}$ be commutative subspace lattices such that $\mathcal{L}_{1} \otimes \mathcal{L}_{2}$ is synthetic. Then $\operatorname{alg}\left(\mathcal{L}_{1} \otimes \mathcal{L}_{2}\right)=\operatorname{alg} \mathcal{L}_{1} \otimes \operatorname{alg} \mathcal{L}_{2}$.

Proof. Let $\mathcal{A}_{1}=\operatorname{alg} \mathcal{L}_{1}$ and $\mathcal{A}_{2}=\operatorname{alg} \mathcal{L}_{2}$. By Theorem 4.2, $\operatorname{lat}\left(\mathcal{A}_{1} \otimes\right.$ $\left.\mathcal{A}_{2}\right)=\mathcal{L}_{1} \otimes \mathcal{L}_{2}$ while, by Theorem 2.1, $\operatorname{lat} \operatorname{alg}\left(\mathcal{L}_{1} \otimes \mathcal{L}_{2}\right)=\mathcal{L}_{1} \otimes \mathcal{L}_{2}$. Since $\mathcal{L}_{1} \otimes \mathcal{L}_{2}$ is synthetic, $\operatorname{alg}\left(\mathcal{L}_{1} \otimes \mathcal{L}_{2}\right)=\mathcal{A}_{1} \otimes \mathcal{A}_{2}$. 


\section{REFERENCES}

[1] W. B. Arveson, Operator algebras and invariant subspaces, Annals of Math. (2) 100 (1974), 433-532.

[2] F. Gilfeather, A. Hopenwasser and D. R. Larson, Reflexive algebras with finite width lattices: tensor products, cohomology, compact perturbations, J. Funct. Anal. 55 (1984), 176-199.

[3] F. Gilfeather and D. R. LARson, Structure in reflexive subspace lattices, J. London Math. Soc. (2) 26 (1982), 117-131.

[4] P. R. Halmos, Reflexive lattices of subspaces, J. London Math. Soc. (2) 4 (1971), 257-263.

[5] K. J. HARRISON, The tensor product formula for reflexive subspace lattices, Canad. Math. Bull. 38 (1995), 308-316.

[6] A. Hopenwasser, Tensor products of reflexive subspace lattices, Mich. Math. J. 31 (1984), 359-370.

[7] A. Hopenwasser And J. Kraus, Tensor products of reflexive algebras II, J. London Math. Soc. (2) 28 (1983), 359-362.

[8] E. G. Katsoulis, Reflexivity for a class of subspace lattices, Math. Proc. Cambridge Phil. Soc. 119 (1996), 67-71.

[9] J. Kraus, The slice map problem and approximation properties, J. Funct. Anal. 102 (1991), 116-155.

[10] M. S. Lambrou and W. E. LongstafF, Non-reflexive pentagon subspace lattices, Studia Math. 125 (1997), 187-199.

[11] J. R. Ringrose, On some algebras of operators, Proc. London Math. Soc. (3) 15 (1965), 61-83.

[12] V. S. Shulman and I. G. Todorov, On subspace lattices I. Closedness type properties and tensor products, Int. Eq. Operator Th., to appear.

[13] D. Symes, Structure of doubly generated reflexive lattices, Quart. J. Math. Oxford Ser. (2) 49 (1998), 229-235.

Ivan G. Todorov,

Department of Pure Mathematics,

Queen's University Belfast,

Belfast BT7 1NN,

N. Ireland

i.todorov@qub.ac.uk

Received on 8 March 2004. 